\title{
On the harmonic oscillator on the Lobachevsky plane
}

\author{
P. Šťovíček, M. Tušek
}

\begin{abstract}
Department of Mathematics, Faculty of Nuclear Science, Czech Technical University, Trojanova 13, 12000 Prague, Czech Republic
\end{abstract}

\begin{abstract}
We introduce the harmonic oscillator on the Lobachevsky plane with the aid of the potential $V(\varrho)=\left(a^{2} \omega^{2} / 4\right) \sinh (\varrho / a)^{2}$ where $a$ is the curvature radius and $\varrho$ is the geodesic distance from a fixed center. Thus the potential is rotationally symmetric and unbounded likewise as in the Euclidean case. The eigenvalue equation leads to the differential equation of spheroidal functions. We provide a basic numerical analysis of eigenvalues and eigenfunctions in the case when the value of the angular momentum, $m$, equals 0 .
\end{abstract}

\section{Introduction}

This short contribution is dedicated to the memory of Vladimir Geyler, our dear colleague and friend who, to our great sorrow, passed recently and very unexpectedly away. Among his numerous scientific activities in the framework of mathematical physics one can point out his interest in the influence of nontrivial geometries on the properties of quantum mechanical systems which was very intensive especially in the last period. Let us mention just a few of his results focused on this sort of problems: the effect of surface curvature on magnetic moment and persistent currents [6], the general form of on-diagonal singularities of the Green function for Schrödinger operators on curved spaces [5], and zero modes in a system of Aharonov-Bohm solenoids on the Lobachevsky plane [9].

In one of the very last scientific contributions due to Vladimir Geyler [10], on which we had the pleasure to collaborate, a quantum dot with impurity in the Lobachevsky plane is discussed. This paper was intended as a step towards an extension to the hyperbolic plane of results derived in [4] where a similar problem is treated in the case of the Euclidean geometry. The current article aims to complete the discussion from [10] by a short numerical analysis of spectral properties of the harmonic oscillator on the Lobachevsky plane. In particular, we are interested in the effect of the surface curvature on these properties. 
It has been observed already some time ago that various solvable quantum mechanical systems admit an explicit treatment also in the case of hyperbolic geometry, though with interesting nontrivial modifications (see, for example, [8, 1]). Also the spectral problem for the quantum harmonic oscillator on the Lobachevsky plane, as we introduce it below, leads to a differential equation which is well known from the theory of special functions, namely to the differential equation of spheroidal functions. It should be emphasized, however, that the history of spheroidal functions is much more recent than that of more traditional special functions like Bessel functions or Legendre polynomials. For example, one of the basic monographs devoted to these functions appeared only in the fifties of the last century [11]. Also the notation is still not fully uniform. One can compare, for example, 11] with [2]. Here we follow the latter source. Furthermore, there are cases of values of parameters on which the spheroidal functions depend that have not been fully investigated. In this connection we note that particularly the values of parameters which are of interest for our model are treated in the textbooks in a rather marginal way. Also effective numerical algorithms to evaluate the spheroidal functions seem to be rather tedious to create and not available for all cases.

These circumstances make the numerical and qualitative analysis of the spectrum more complicated than one might expect at first glance. The numerical results presented in this contribution were derived with the aid of the computer algebra system Mathematica. On the contrary to other "more traditional" special functions, the spheroidal functions were implemented in this system only in the version which was the very last one at the time of this writing.

\section{The Hamiltonian}

Denote by $a, 0<a<\infty$, the so called curvature radius which is related to the scalar curvature by the formula $R=-2 / a^{2}$, and by $(\varrho, \phi), 0<\varrho<\infty, 0 \leq \phi<2 \pi$, the geodesic polar coordinates on the Lobachevsky plane. Taking the mass equal $1 / 2$ and $\hbar=1$, the Hamiltonian has the form

$$
H=-\left(\frac{\partial^{2}}{\partial \varrho^{2}}+\frac{1}{a} \operatorname{coth}\left(\frac{\varrho}{a}\right) \frac{\partial}{\partial \varrho}+\frac{1}{a^{2} \sinh \left(\frac{\varrho}{a}\right)^{2}} \frac{\partial^{2}}{\partial \phi^{2}}+\frac{1}{4 a^{2}}\right)+V(\varrho),
$$

and is defined in $L^{2}\left((0, \infty) \times S^{1}, a \sinh (\varrho / a) \mathrm{d} \varrho \mathrm{d} \phi\right)$ on its natural domain. Here $V(\varrho)$ is the potential of the harmonic oscillator on the hyperbolic plane. Its choice is ambiguous. Of course, one requires that in the flat limit, $a \rightarrow \infty, V(\rho)$ becomes the usual potential of the isotropic harmonic oscillator on the Euclidean plane, $V^{\infty}(\varrho)=\omega^{2} \varrho^{2} / 4$. At least three choices of $V(\varrho)$ have been discussed in the literature so far.

A distinguished possibility is

$$
V_{1}(\varrho)=\frac{1}{4} a^{2} \omega^{2} \tanh \left(\frac{\varrho}{a}\right)^{2} .
$$

since in that case one gets a Hamiltonian with a comparatively rich symmetry, a so called super-integrable model. this is demonstrated in detail on the level of classical 
mechanics in [12], see also [13]. Notice that this potential is bounded and thus the spectrum of the corresponding quantum Hamiltonian contains an absolutely continuous part, the interval $\left[E_{0}(a), \infty[\right.$. The spectral analysis for this case is covered by paper [6]. It turns out that $E_{0}(a) \sim a^{2}$ as $a \rightarrow \infty$, and so the continuous part disappears in the flat limit. Moreover, as expected, the number of eigenvalues in the curved case is finite but tends to infinity in the flat limit, and the eigenvalues approach the discrete spectrum of the flat Hamiltonian.

Further, a nonlinear quantum model with a potential of the type

$$
V_{2}(\varrho)=\text { const } \frac{\varrho^{2}}{1+\lambda \varrho^{2}}
$$

depending on a parameter $\lambda$ is discussed in [7]. The treatment of this model also relies heavily on symmetry properties which allow for explicit solutions.

In the current paper we stick, however, to a choice made in [10] and otherwise not discussed in the above cited papers. We set

$$
V(\varrho)=\frac{1}{4} a^{2} \omega^{2} \sinh \left(\frac{\varrho}{a}\right)^{2} .
$$

With this choice, the potential is unbounded, likewise as in the Euclidean case. Consequently, the corresponding Hamiltonian $H$ has a purely discrete spectrum depending on the parameter $a$.

Next we rescale the Hamiltonian letting

$$
\tilde{H}=a^{2} H \text {. }
$$

Substituting $\xi=\cosh (\varrho / a)$ we obtain

$$
\tilde{H}=\left(1-\xi^{2}\right) \frac{\partial^{2}}{\partial \xi^{2}}-2 \xi \frac{\partial}{\partial \xi}+\left(1-\xi^{2}\right)^{-1} \frac{\partial^{2}}{\partial \phi^{2}}+\frac{a^{4} \omega^{2}}{4}\left(\xi^{2}-1\right)-\frac{1}{4}
$$

in $L^{2}\left((1, \infty) \times S^{1}, \mathrm{~d} \xi \mathrm{d} \phi\right)$. Using the rotational symmetry which amounts to a Fourier transform in the variable $\phi, \tilde{H}$ may be decomposed into a direct sum as follows

$$
\begin{aligned}
& \tilde{H}=\sum_{m=-\infty}^{\infty} \tilde{H}_{m} \\
& \tilde{H}_{m}=-\frac{\partial}{\partial \xi}\left(\left(\xi^{2}-1\right) \frac{\partial}{\partial \xi}\right)+\frac{m^{2}}{\xi^{2}-1}+\frac{a^{4} \omega^{2}}{4}\left(\xi^{2}-1\right)-\frac{1}{4}
\end{aligned}
$$

For all $m \in \mathbb{Z}, \tilde{H}_{m}$ acts in $L^{2}((1, \infty), \mathrm{d} \xi)$.

\section{Eigenvalues and eigenfunctions}

Denote by $H_{m}, m \in \mathbb{Z}$, the restrictions of $H$ to the eigenspace of the angular momentum with value $m$. We prefer to regard $H_{m}$ as an operator in $L^{2}\left(\mathbb{R}_{+}, \mathrm{d} \varrho\right)$. This 
assumes an obvious unitary transform to pass from the measure $a \sinh (\varrho / a) \mathrm{d} \varrho$ in the $L^{2}$ space on $\mathbb{R}_{+}$to the measure $\mathrm{d} \varrho$. On the general grounds, the spectrum of $H_{m}$ is semibounded below, discrete and simple [3, 14]. Set

$$
q=\frac{a^{2} \omega}{2}
$$

If $\tilde{E}_{n}^{m}, n=0,1,2, \ldots$, is the $n$ the eigenvalue of $\tilde{H}_{m}$ then

$$
E_{n}^{m}=\frac{1}{a^{2}} \tilde{E}_{n}^{m}=\frac{\tilde{E}_{n}^{m}}{2 q} \omega
$$

is the $n$th eigenvalue of $H_{m}$.

The eigenvalue equation $\tilde{H}_{m} \psi=E \psi$ written in the coordinate $\xi$ takes the form

$$
\left(1-\xi^{2}\right) \frac{\partial^{2} \psi}{\partial \xi^{2}}-2 \xi \frac{\partial \psi}{\partial \xi}+\left(\lambda+4 \theta\left(1-\xi^{2}\right)-\frac{m^{2}}{1-\xi^{2}}\right) \psi=0
$$

where

$$
\lambda=-E-\frac{1}{4}, 4 \theta=-\frac{a^{2} \omega^{2}}{4}=-q^{2} .
$$

Differential equation (11) is known in the theory of special functions as the differential equation of spheroidal functions [2, 11]. The notation used below follows the source [2]. All parameters in (11) are in general complex numbers. There are two solutions that behave like $\xi^{\nu}$ times a single-valued function and $\xi^{-\nu-1}$ times a single-valued function at $\infty$. The exponent $\nu$ is a function of $\lambda, \theta, m$, and is called the characteristic exponent. Usually, it is more convenient to regard $\lambda$ as a function of $\nu, m$ and $\theta$. We shall write $\lambda=\lambda_{\nu}^{m}(\theta)$. The functions $\lambda_{\nu}^{m}(\theta)$ obey the symmetry relations

$$
\lambda_{\nu}^{m}(\theta)=\lambda_{\nu}^{-m}(\theta)=\lambda_{-\nu-1}^{m}(\theta)=\lambda_{-\nu-1}^{-m}(\theta) .
$$

A first group of solutions (the so called radial spheroidal functions) is obtained as expansions in series of Bessel functions. They are denoted $S_{\nu}^{m(j)}(\xi, \theta), j=1,2,3,4$. Several relations between the radial spheroidal functions are known, notably

$$
S_{\nu}^{m(3)}(\xi, \theta)=S_{\nu}^{m(1)}(\xi, \theta)+i S_{\nu}^{m(2)}(\xi, \theta)=-i e^{-i \nu \pi} S_{-\nu-1}^{m(3)}(\xi, \theta)
$$

and

$$
S_{\nu}^{m(3)}(\xi, \theta)=\frac{1}{i \cos (\nu \pi)}\left(S_{-\nu-1}^{m(1)}(\xi, \theta)+i e^{-i \nu \pi} S_{\nu}^{m(1)}(\xi, \theta)\right) .
$$

Also the asymptotic behavior of radial spheroidal functions is well known. It turns out that among these functions the only one which is square integrable near infinity is $S_{\nu}^{m(3)}(\xi, \theta)$. For $q>0$ it holds

$$
S_{\nu}^{m(3)}\left(\xi,-\frac{q^{2}}{4}\right)=-\frac{1}{q \xi} \exp \left(-q \xi-i \frac{\nu \pi}{2}\right)\left(1+O\left(\xi^{-1}\right)\right)
$$

as $\xi \rightarrow+\infty$. 
To control the behavior near the singular point $\xi=1$ one uses expansions in series in Legendre functions. These solutions are called angular spheroidal functions and are denoted $P s_{\nu}^{m}(\xi, \theta)$ and $Q s_{\nu}^{m}(\xi, \theta)$. There exist relations between these two types of spheroidal functions. In particular, it holds

$$
S_{\nu}^{m(1)}(\xi, \theta)=V_{\nu}^{m}(\theta) Q s_{-\nu-1}^{m}(\xi, \theta)
$$

where $V_{\nu}^{m}(\theta)$ is a coefficient independent of $\xi$. Consequently,

$$
S_{\nu}^{m(3)}(\xi, \theta)=\frac{1}{i \cos (\nu \pi)}\left(V_{-\nu-1}^{m}(\theta) Q s_{\nu}^{m}(\xi, \theta)+i e^{-i \nu \pi} V_{\nu}^{m}(\theta) Q s_{-\nu-1}^{m}(\xi, \theta)\right) .
$$

The angular spheroidal functions asymptotically behave, as $\xi \rightarrow+\infty$, in the following way:

$$
P s_{\nu}^{m}(\xi, \theta)=\frac{1}{\Gamma(1-m) s_{\nu}^{m}(\theta)}\left(\frac{\xi-1}{2}\right)^{-m / 2}(1+O(\xi-1))
$$

where $s_{\nu}^{m}(\theta)$ is some coefficient (for its definition we refer to [2]). Particularly, we have

$$
\frac{1}{s_{\nu}^{0}(\theta)}=P s_{\nu}^{0}(1, \theta)
$$

Furthermore,

$$
Q s_{\nu}^{0}(\xi, \theta)=-\frac{1}{2 s_{\nu}^{0}(\theta)} \log (\xi-1)+O(1)
$$

and

$$
(\xi-1) \frac{\partial Q s_{\nu}^{0}(\xi, \theta)}{\partial \xi}=-\frac{1}{2 s_{\nu}^{0}(\theta)}+o(1)
$$

It follows that

$$
S_{\nu}^{0(3)}(\xi, \theta)=-\frac{1}{2 i \cos (\nu \pi)}\left(\frac{V_{-\nu-1}^{0}(\theta)}{s_{\nu}^{0}(\theta)}+i e^{-i \nu \pi} \frac{V_{\nu}^{0}(\theta)}{s_{-\nu-1}^{0}(\theta)}\right) \log (\xi-1)+O(1) .
$$

We conclude that $\tilde{\psi}(\xi)=S_{\nu}^{m(3)}\left(\xi,-q^{2} / 4\right)$ is an eigenfunction and, at the same time, $\tilde{E}_{\nu}^{m}=-\lambda_{\nu}^{m}\left(-q^{2} / 4\right)-1 / 4$ is an eigenvalue of $\tilde{H}_{m}$ if and only if $\tilde{\psi}(\xi)$ has no singularity as $\xi \rightarrow 1+$. Further we restrict ourselves to the case $m=0$. The function $\lambda_{\nu}^{0}(\theta)$ is real for $\nu=-1 / 2+i t, t \in \mathbb{R}$, and becomes negative if $t$ is sufficiently large. The eigenvalues of $\tilde{H}_{0}$ are the numbers $-\lambda_{-1 / 2+i t}^{0}\left(-q^{2} / 4\right)-1 / 4$ where $t$ solves the equation

$$
e^{-t \pi / 2} \frac{V_{-1 / 2-i t}^{0}\left(-q^{2} / 4\right)}{s_{-1 / 2+i t}^{0}\left(-q^{2} / 4\right)}-e^{t \pi / 2} \frac{V_{-1 / 2+i t}^{0}\left(-q^{2} / 4\right)}{s_{-1 / 2-i t}^{0}\left(-q^{2} / 4\right)}=0 .
$$

Because of symmetry (2) it suffices to consider $t>0$. Equivalently, in the numerical computations the following form of this equation proved itself to be rather effective:

$$
\lim _{\xi \rightarrow 1+}(\xi-1) \frac{\partial S_{-1 / 2+i t}^{0(3)}\left(\xi,-q^{2} / 4\right)}{\partial \xi}=0
$$


Finally, we present a few numerical results. Figure1 depicts several first eigenvalues of $H_{0}$. More precisely, the figure contains plots of eigenvalues $E_{n}^{0}(q) / \omega=\tilde{E}_{n}^{0}(q) /(2 q)$ for $n=0,1,2$. These functions of the variable $q=a^{2} \omega / 2$ decrease monotonically, and in the limit $q \rightarrow \infty$ they approach the eigenvalues in the flat case, namely $2 n+1$. On the other hand, for $q \rightarrow 0+$ the eigenvalues tend to infinity.

Next we plot the corresponding eigenfunctions for several values of the parameter $q$. We have chosen $q=0.5,5, \infty$. The last value corresponds to the flat case. To reach this goal it was necessary to fix the value of $\omega$. We have taken $\omega=1$. Then the chosen values of $q$ correspond to the curvature radius $a^{2}=1,10, \infty$. An eigenfunction $\tilde{\psi}(\xi)$ of $\tilde{H}_{0}$ equals $S_{\nu}^{0(3)}\left(\xi,-q^{2} / 4\right)$ times a normalization factor for a suitable value of $\nu \in-1 / 2+i \mathbb{R}_{+}$. The plotted eigenfunctions are expressed in the variable $\varrho$. Thus the transformed eigenfunction of $H_{0}$ in $L^{2}\left(\mathbb{R}_{+}, \mathrm{d} \varrho\right)$ equals

$$
\psi(\varrho)=\left(\frac{1}{a} \sinh \left(\frac{\varrho}{a}\right)\right)^{1 / 2} \tilde{\psi}\left(\cosh \left(\frac{\varrho}{a}\right)\right) .
$$

The fact that we stick to the same Hilbert space in all cases, namely to $L^{2}\left(\mathbb{R}_{+}, \mathrm{d} \varrho\right)$, facilitates the comparison of eigenfunctions for various values of the curvature radius. The same Hilbert space is used also in the limiting Euclidean case when $a=\infty$. The eigenfunctions for the flat case are, of course, well known from textbooks. Let us recall that in the Euclidean plane we have

$$
\psi_{n}^{0}(\varrho)=(-1)^{n} \sqrt{\varrho} L_{n}^{0}\left(\frac{\varrho^{2}}{2}\right) \exp \left(-\frac{\varrho^{2}}{4}\right) .
$$

Looking at figures 2, 3 and 4 depicting the eigenfunctions indexed $n=0,1$ and 2, respectively, one observes clearly that a more curved space (corresponding to smaller a) forces the eigenfunctions to concentrate more closely to the center of the harmonic oscillator.

\section{Acknowledgments}

One of the authors (P. Š. ) wishes to acknowledge gratefully a partial support from grant No. 201/05/0857 of the Grant Agency of Czech Republic.

\section{References}

[1] M. Antoine, A. Comtet and S. Ouvry, Scattering on a hyperbolic torus in a constant magnetic field, J. Phys. A: Math. Gen. 23 (1990), 3699-3710.

[2] H. Bateman and A. Erdélyi, Higher Transcendental Functions III. McGraw-Hill Book Company, 1955.

[3] F. A. Berezin, and M. A. Shubin, The Schrödinger Equation. Kluwer Academic Publishers, 1991. 
[4] J. Brüning, V. Geyler and I. Lobanov, Spectral properties of a short-range impurity in a quantum dot, J. Math. Phys. 46 (2004), 1267-1290.

[5] J. Brüning, V. Geyler and K. Pankrashkin, On-diagonal singularities of the Green function for Schrödinger operators, J. Math. Phys. 46 (2005), 113508.

[6] D. V. Bulaev, V. A. Geyler and V. A. Margulis, Effect of surface curvature on magnetic moment and persistent currents in the two-dimensional quantum ring and dots, Phys. Rev. B 69 (2004), 195313.

[7] J. F. Cariñena, M. F. Rañada and M. Santander, The quantum harmonic oscillator on the sphere and the hyperbolic plane, Ann. Physics 322 (2007), 2249-2278.

[8] A. Comtet, On the Landau levels on the hyperbolic plane, Ann. Physics 173 (1987), 185-209.

[9] V. A. Geyler and P. Šťvíček, Zero modes in a system of Aharonov-Bohm solenoids on the Lobachevsky plane, J. Phys. A: Math. Gen. 39 (2006), 1375-1384.

[10] V. A. Geyler and P. Šťovíček and M. Tušek, A quantum dot with impurity in the Lobachevsky plane, (2007) arXiv:math-ph/0709.2790.

[11] J. Meixner and F.V. Schäfke, Mathieusche Funktionen und Sphäroidfunktionen. Springer-Verlag, 1954.

[12] M. F. Rañada and M. Santader, On harmonic oscillators on the two-dimensional sphere $S^{2}$ and the hyperbolic plane $H^{2}$, J. Math. Phys. 43 (2002), 431-451.

[13] M. F. Rañada and M. Santader, On harmonic oscillators on the two-dimensional sphere $S^{2}$ and the hyperbolic plane $H^{2}$. II., J. Math. Phys. 44 (2003), 2149-2167.

[14] J. Weidman, Linear Operators in Hilbert Spaces. Springer, 1980. 


\section{Figure captions}

FIGURE1, Plots of $E_{n}^{0}(q) / \omega=\tilde{E}_{n}^{0}(q) /(2 q), n=0,1,2$, where $E_{n}^{0}$ is the $n$th eigenvalue of $H_{0}$ and $q=a^{2} \omega / 2$.

FIGURE 2, The normalized eigenfunction of $H_{0}, \psi_{0}^{0}(\varrho) \in L^{2}\left(\mathbb{R}_{+}, \mathrm{d} \varrho\right)$, for the values $\omega=1$ and $a^{2}=1$ (solid), $a^{2}=10$ (dashed), $a^{2}=\infty$ (= the flat case, dotted).

FIGURE 3. The normalized eigenfunction of $H_{0}, \psi_{1}^{0}(\varrho) \in L^{2}\left(\mathbb{R}_{+}, \mathrm{d} \varrho\right)$, for the values $\omega=1$ and $a^{2}=1$ (solid), $a^{2}=10$ (dashed), $a^{2}=\infty$ (= the flat case, dotted).

FIGURE 4. The normalized eigenfunction of $H_{0}, \psi_{2}^{0}(\varrho) \in L^{2}\left(\mathbb{R}_{+}, \mathrm{d} \varrho\right)$, for the values $\omega=1$ and $a^{2}=1$ (solid), $a^{2}=10$ (dashed) and $a^{2}=\infty$ (= the flat case, dotted). 


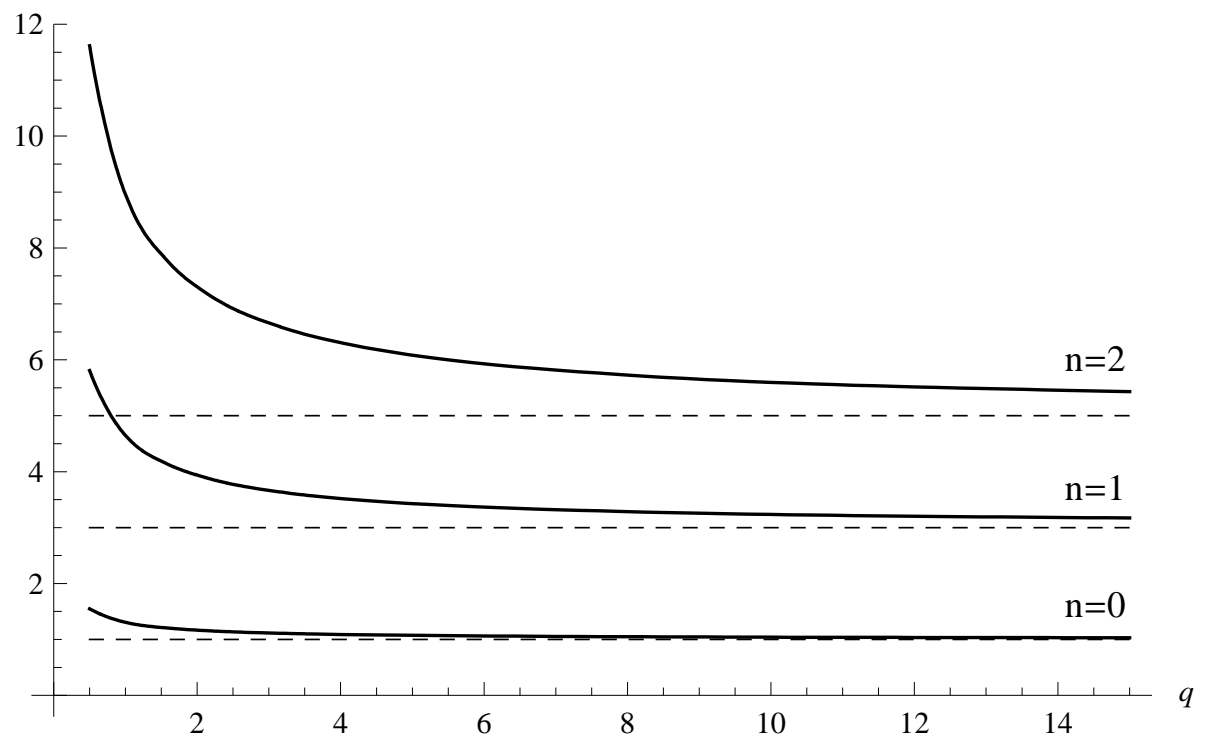

Figure 1: 


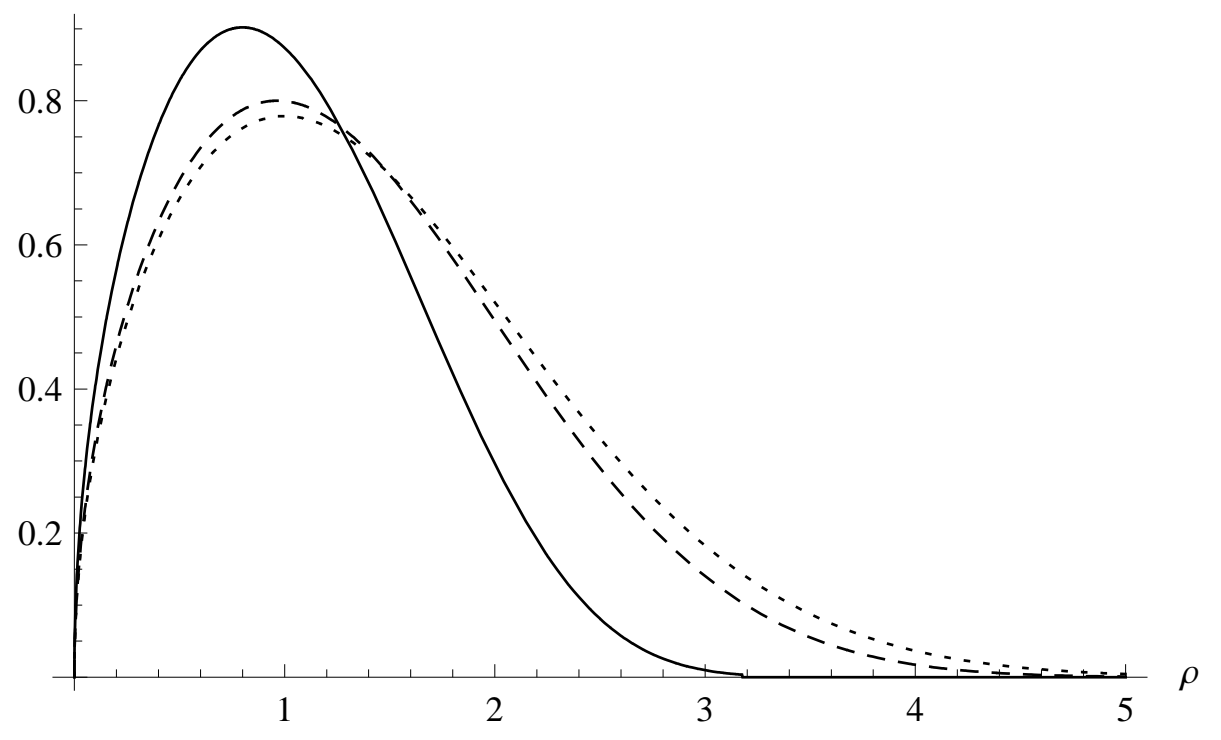

Figure 2: 


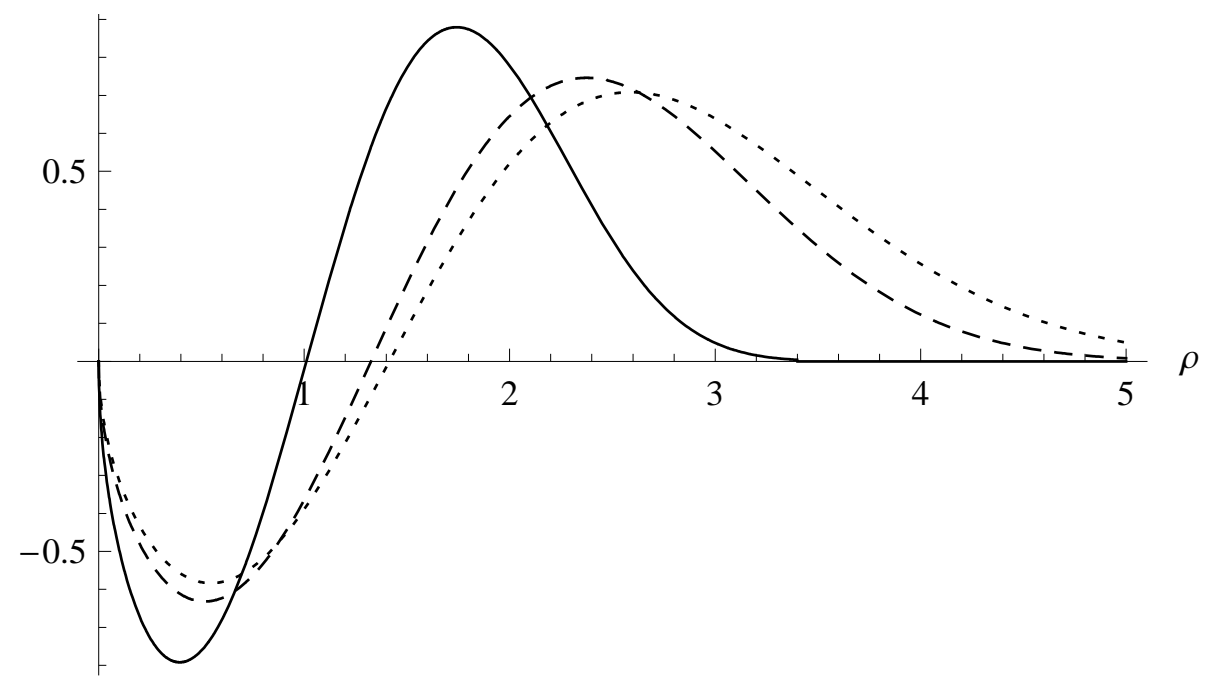

Figure 3: 


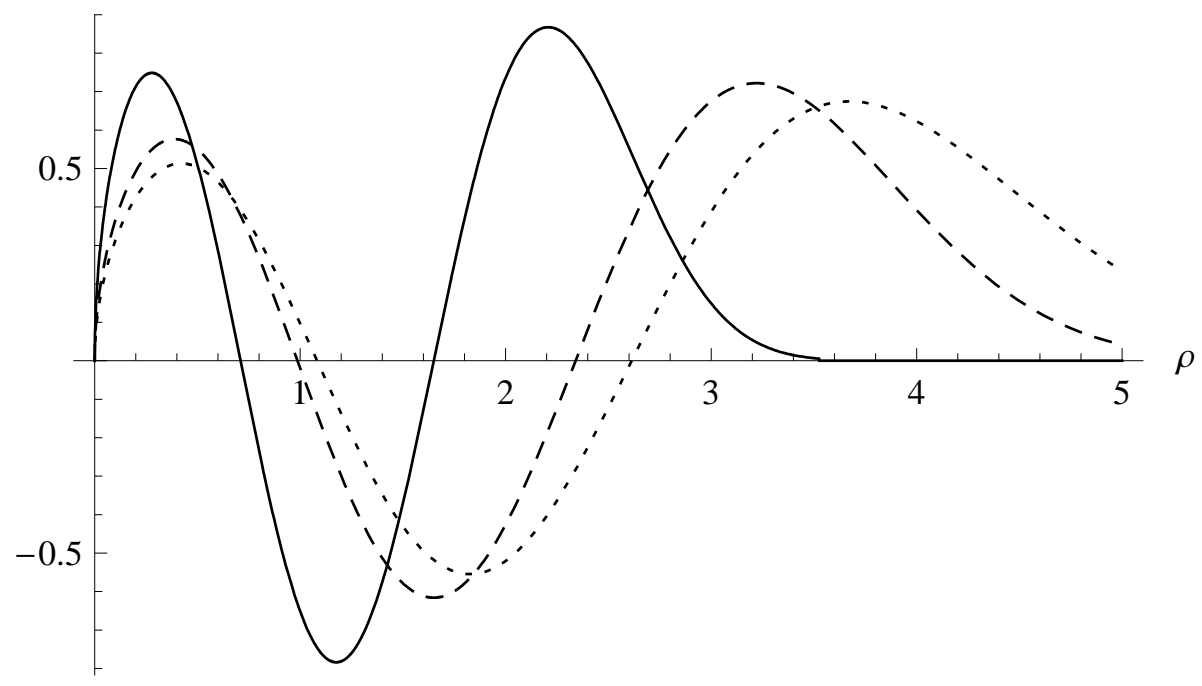

Figure 4: 